\title{
Factors Associated with Nosocomial Bloodstream Infection Caused by Carbapenem-Resistant Klebsiella pneumoniae and With Mortality, from a Single Center
}

\author{
Xiaomei Chen*, Jing Wang, Yuan Li, Jianning Zhang, Hui Han and Hao Wang
}

Department of Critical Care Medicine, Qilu Hospital of Shandong University, China

${ }^{*}$ Corresponding author: Xiaomei Chen, Department of Critical Care Medicine, Qilu Hospital of Shandong University, China, Tel: 8618560081002; Email: chenxm008@sdu.edu.cn

Received date: August 14, 2017; Accepted date: September 01, 2017; Published date: September 08, 2017

Citation: Chen X, Wang J, Li W, Zhang J, Han H, et al. (2017) Factors associated with Nosocomial Bloodstream Infection Caused by CarbapenemResistant Klebsiella pneumoniae and with Mortality, from a Single Center. J Prev Infect Cntrol. Vol.3 No.2:8.

\section{Abstract}

Carbapenem-resistant Klebsiella pneumoniae (CRKP) is an emerging multi-drug nosocomial pathogen. We compared factors associated with bloodstream infection (BSI) caused by CRKP and carbapenem-sensitive $K$. pneumoniae (CSKP) and risk factors associated with mortality. A retrospective cohort design was conducted by our tertiary-care university teaching hospital with 3000 beds. All adults with CRKP and CSKP BSI from January 2010 to December 2016 were included. CRKP and CSKP BSI isolated from blood culture were identified retrospectively in the microbiology laboratory. Demographic and clinical characteristics were collected from electronic medical records. Multivariate analysis was used to examine risk factors of mortality, estimating odds ratios (ORs) and 95\% confidence intervals (Cls). This study included 140 patients, 29 with CRKP BSI and 111 with CRSP BSI. Patients with CRKP BSI had undergone more invasive procedures, tended to have more comorbidities measured by the Charlson index, and had higher rates of previous antimicrobial exposure than those with CRSP BSI. The crude and attributable mortality at 4 weeks for the 29 patients with CRKP BSI was $41.4 \%$ and $25.9 \%$, respectively. On multivariate analysis, factors associated with increased mortality with CRKP BSI were use of ventilation (OR 13.6, 95\% $\mathrm{Cl} 3.0-62.3$ ) and tigecycline treatment (110.5, 5.8-2096.2) and appropriate antibiotics empirical therapy was associated with decreased mortality (0.01, 0.001-0.086). Summary, risk factors for CRKP BSIrelated mortality were ventilation, tigecycline treatment and inappropriate antibiotics empirical therapy. The higher mortality associated with CRKP than CSKP BSI may be mediated by failure to provide effective therapy.

Keywords: Carbapenem-resistant; Klebsiella pneumonia; Multi-drug resistance; Bloodstream infection; Risk factor; Outcome

\section{Introduction}

Infections caused by carbapenem-resistant Klebsiella pneumoniae (CRKP) are associated with high treatment failure and mortality $[1,2]$. These poor outcomes are likely multifactorial in etiology, including greater severity of underlying illness, delays in initiating effective therapy, and lack of effective prophylactic and empiric antibiotics therapy. Identification of risk factors of infection with CRKP may help in therapeutic decision-making and may assist in the early implementation of appropriate infection control measures [3].

Carbapenem-resistant Enterobacteriaceae (CRE) infection was first described in the early 1990s [4] and found to confer broad resistance to most $\beta$-lactam antibiotics including "last-line" carbapenems. The prevalence of CRE infections has become a substantial global health problem, especially in healthcare settings. CRE infection has been recognized by the US Centers for Disease Control and Prevention as an urgent public health threat.

Here, we aimed to determine factors associated with CRKP bacteremia and risk factors of mortality by comparison with carbapenem-sensitive $K$. pneumoniae (CSKP) infection in our institution.

\section{Methods}

We conducted a 6 year retrospective cohort study of 140 patients with $K$. pneumoniae bacteremia from January 2010 to December 2016 in a 3000 bed tertiary care university teaching hospital, Qilu Hospital, in China.

\section{Study population}

Participants were identified among adult inpatients at the hospital for which blood cultures yielded $K$. pneumoniae in the clinical microbiology laboratory database. Only the first episode of $K$. pneumoniae isolation was studied.

Clinical data were collected from patient medical records and included demographic characteristics, comorbidities, Charlson comorbidity index, interventions as well as intensive care unit (ICU) admission. Data were collected on all Gram-negative antibiotics treatments administered during hospitalization before the diagnosis of invasive $K$. pneumoniae infection. Data were also collected on antibiotics administered after the isolation of $K$. pneumoniae and the time to administration of antibiotics with in vitro activity. 
The onset of bacteremia was defined as the date when the first positive blood culture was obtained. Empirical therapy indicates that antibiotics therapy was initiated before in vitro antibiotics susceptibility test results were released by the clinical microbiology laboratory.

\section{Microbiological methods}

Blood cultures were processed by using an automated blood culture system (Bactec 9240; Becton Dickinson). The isolated organisms that grew on culture were identified according to routine bacteriological procedures (Vitek 2 Compact, bioMerieux, France). The laboratories determined the minimum inhibitory concentration (MIC) of imipenem for the $K$. pneumoniae isolates. CRKP was defined as MIC $>16 \mu \mathrm{g} / \mathrm{ml}$ for imipenem; and CSKP was defined as MIC $\leq 1 \mu \mathrm{g} / \mathrm{ml}$ for imipenem. No patients had intermediate MIC values (1-16 $\mu \mathrm{g} / \mathrm{ml}$ ) in accordance with the Clinical and Laboratory Standards Institute criteria.

\section{Statistical analysis}

Data are described with number (\%) or median (range). Continuous variables were compared by Student $t$ test for normally distributed variables and Mann-Whitney $U$ test for non-normally distributed variables. Chi-square or Fisher's exact test was used to compare categorical variables. Multivariate regression analysis was used to determine risk factors associated with mortality, estimating odds ratios (ORs) and $95 \%$ confidential intervals (Cls). Survival curves were created by Kaplan-Meier estimation. $\mathrm{P}<0.05$ was considered statistically significant. All analyses involved use of SPSS 22.0 (SPSS Inc., Chicago, IL).

\section{Results}

\section{Study population}

During the study period, 140 patients had a bloodstream infection due to $K$. pneumoniae: 29 (20.7\%) with CRKP and 111 (79.3\%) with CSKP. CRKP and CSKP patients had similar median age (64 vs. 58 years), with similar distribution of sex (93.1\% vs. 91.1\% males) (Table 1). The 2 groups were similar in Charlson comorbidity index. However, the frequency of intensive care unit (ICU) admissions was significantly higher for CRKP than CSKP patients (48.3\% vs. $14.4 \%)$, as was the frequency of some other malignancies. It was higher for insertion of central venous catheters $(65.5 \%$ vs. $32.4 \%)$ and urinary catheters $(58.6 \%$ vs. $26.1 \%)$ and ventilation $(79.3 \%$ vs. $18 \%)$. CRKP patients underwent significantly more invasive procedures during hospitalization than CSKP patients.

Table 1 Bivariate analysis of factors associated with carbapenem-resistant Klebsiella pneumoniae (CRKP) and carbapenemsusceptible K. pneumoniae (CSKP) bloodstream infection. ICU: Intensive Care Unit; OR: Odds Ratio; 95\% Cl: 95\% Confidence Interval.

\begin{tabular}{|c|c|c|c|c|}
\hline Factors & CRKP (n=29) & $\operatorname{CSKP}(n=111)$ & OR & $P$ value \\
\hline Age, year (median) & 64 & 58 & & 0.143 \\
\hline Sex, male, n (\%) & 27 (93.1) & $102(91.9)$ & $1.2(0.2-5.8)$ & 0.829 \\
\hline ICU admission (\%) & $14(48.3)$ & $16(14.4)$ & $5.5(2.3-13.6)$ & $<0.001$ \\
\hline Hospitalization in previous 3 months, $\mathrm{n}(\%)$ & $0(0)$ & $14(12.6)$ & $0.1(0.01-1.9)$ & 0.135 \\
\hline Charlson comorbidity index $\geq 3$ & $8(27.6)$ & $31(27.9)$ & $1.0(0.4-2.5)$ & 0.971 \\
\hline \multicolumn{5}{|l|}{ Co-morbidities, n (\%) } \\
\hline Diebetes mellitus & $8(27.6)$ & $27(24.3)$ & $1.2(0.5-3.0)$ & 0.718 \\
\hline Chemotherapy & $3(10.3)$ & $17(15.3)$ & $0.6(0.2-2.3)$ & 0.499 \\
\hline Cardiovascular disease & $10(34.5)$ & $18(16.2)$ & $2.7(1.1-6.8)$ & 0.031 \\
\hline Chronic lung disease & $1(3.5)$ & $2(1.8)$ & $1.9(0.2-22.2)$ & 0.592 \\
\hline Chronic kidney disease & $4(13.8)$ & $0(0)$ & $39.3(2.1-754.4)$ & 0.015 \\
\hline Leukemia or lymphoma & $2(6.9)$ & $18(16.2)$ & $0.4(0.1-1.8)$ & 0.216 \\
\hline Other malignancy & $2(6.9)$ & $32(28.8)$ & $0.2(0.1-0.8)$ & 0.026 \\
\hline \multicolumn{5}{|l|}{ Invasive procedure, n (\%) } \\
\hline Surgery & $7(24.1)$ & $20(18.0)$ & $1.4(0.5-3.9)$ & 0.459 \\
\hline Central venous catheter & $19(65.5)$ & $36(32.4)$ & $4.0(1.7-9.4)$ & 0.002 \\
\hline Urinary catheter & $17(58.6)$ & $29(26.1)$ & $3.3(1.4-7.5)$ & 0.006 \\
\hline Ventilation & $23(79.3)$ & $20(18.0)$ & $17.4(6.3-48.4)$ & 0.001 \\
\hline
\end{tabular}




\begin{tabular}{|c|c|c|c|c|}
\hline Carbapenem & $13(44.8)$ & $22(19.8)$ & $3.3(1.4-7.8)$ & 0.007 \\
\hline Tigecycline & $9(31.0)$ & $1(0.9)$ & $49.5(5.9-412.5)$ & 0.001 \\
\hline Piperacillin-tazobactam & $6(20.7)$ & $10(9.0)$ & $0.3(0.1-0.7)$ & 0.012 \\
\hline Floxacillin & $0(0)$ & $14(12.6)$ & $0.1(0.0-2.0)$ & 0.135 \\
\hline Fluoroquinolones & $6(20.7)$ & $19(17.1)$ & $1.3(0.5-3.5)$ & 0.655 \\
\hline Cephalosporin & $13(44.8)$ & $20(18.0)$ & $3.7(1.5-8.9)$ & 0.004 \\
\hline Appropriate antibiotics empirical therapy, $\mathrm{n}(\%)$ & $6(20.7)$ & $92(82.9)$ & $0.1(0.0-0.2)$ & 0.001 \\
\hline
\end{tabular}

The previous use of carbapenem, tigecycline, pipercillintazobactam and cephalosporin was significantly related with CRKP than CSKP BSI. The frequency of appropriate antibiotics empirical therapy was greater with CSKP than CRKP BSI. Floxacillin as first-line antibiotics treatment was less associated with CRKP than CSKP BSI, although not significantly.

Among the 140 patients, $10.9 \%$ died within 1 week after the isolation of $K$. pneumoniae and $25.9 \%$ died within 4 weeks. The crude mortality rates were higher at weeks 1 and 4 for CRKP than CSKP patients. On multivariate analysis, factors associated with increased mortality with CRKP BSI were use of ventilation (OR 13.6, 95\% Cl 3.0-62.3) and tigecycline treatment (110.5, 5.8-2096.2) and appropriate antibiotics empirical therapy was associated with decreased mortality $(0.01,0.001-0.086)$ (Table 2).

Table 2 Multivariate analysis of risk factors for 4 week mortality in patients with CRKP and CSKP bloodstream infection.

\begin{tabular}{|l|l|l|}
\hline Parameter & Adjusted OR (95\% CI) & P value \\
\hline Ventilation & $13.6(3.0-62.3)$ & 0.001 \\
\hline Tigecycline treatment & $110.5(5.8-2096.2)$ & 0.002 \\
\hline $\begin{array}{l}\text { Appropriate antibiotics } \\
\text { empirical therapy }\end{array}$ & $0.01(0.001-0.086)$ & 0.001 \\
\hline
\end{tabular}

To plot the outcome of patients with CRKP or CSKP bacteremia, we generated Kalpan-Meier survival curves starting from the date infection was detected. The outcome was significantly worse for CRKP than CSKP patients (Figure 1).

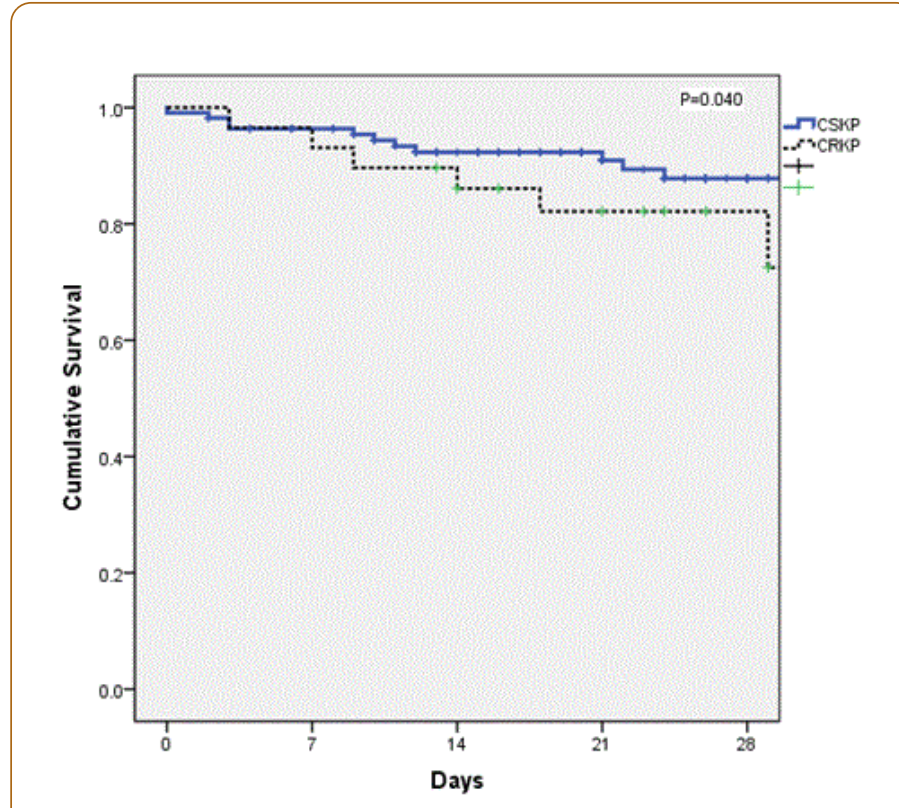

Figure 1 Kaplan-Meier curve analysis of the impact of CRKP and CSKP infection on 4 week mortality.

\section{Discussion}

Our study results suggest that mechanical ventilation, tigecycline and inappropriate antibiotics empirical therapy are crucial factors in determining the outcomes of patients with CRKP bacteremia (Table 3).

Table 3 Crude and attributable mortality of CRKP and CSKP at weeks 1 and 4.

\begin{tabular}{|l|l|l|l|}
\hline Mortality, $\mathbf{n}(\%)$ & CRKP (n=29) & CSKP (n=111) & P value \\
\hline At 1 week & $5(17.2 \%)$ & $7(6.3 \%)$ & 0.073 \\
\hline At 4 week & $12(41.4 \%)$ & $17(15.3 \%)$ & 0.04 \\
\hline
\end{tabular}

During the study period, the rate of carbapenem resistance was $20.7 \%$, especially low in cancer patients, which was not consistent with previous reports. Trecarichi and Tumbarello showed greater than $98 \%$ prevalence of CSKP isolates in cancer patients from 2007 to 2013 [5]. The other study of 13 Italian hematological units reported a rate of CRKP that increased significantly from $21.4 \%$ in 2010 to $75.9 \%$ in 2013 [6]. Neutropenia, HM and chemotherapy/radiation therapy have been recognized as independent risk factors of CRKP infection [7-9].

Our study failed to show an independent effect of CRKP BSI infection with co-morbidities, invasive procedures or previous antimicrobial treatment on mortality. This finding could be due to our relatively small sample size, which would not show all the factors associated with mortality. Diabetes mellitus, chemotherapy, chronic lung disease and cardiovascular disease 
did not differ between the 2 groups, as was previously found [10]

In our study, patients with CRKP BSI more frequently received inappropriate empiric antibiotics therapy than CSKP patients (79.3\% vs. 17.1\%, $P=0.001$ ). Ben-David et al. demonstrated ineffective antimicrobial therapy in all patients with CRKP and CSKP BSI ( $88 \%$ vs. $21 \%)$ associated with increased mortality [11] Daikos et al. also suggested that the effect of carbapenem resistance on mortality was probably mediated by the failure to provide effective antimicrobial therapy in patients with CRKP or CSKP infection (68\% vs. 32\%; OR 2.27, 95\% Cl 0.91-5.64) [12]. Although one study showed that tigecycline treatment appeared to be suitable for BSI caused by carbapenem-resistant Gramnegative bacteria [13], our study suggested that tigecycline could be associated with 100 -fold risk of acquiring CRKP. To explore the phenomenon, we found that patients with previous infection caused by other carbapenem-resistant Gram-negative bacteria had received tigecycline. The optimal treatment of CRKP bacteremia remains unknown. Because the isolation of CRKP is a new phenomenon in our hospital, the power of both risk factors and outcome analyses is limited. From previous studies and experiences, strict infection control measures must be implemented to prevent further spread of CRKP.

In our study, malignancy was less frequent with CRKP than CSKP BSI. However, Messina et al. suggested that patients with malignant tumors were at increased risk of readmission with CRKP infection [14], which has been linked to microbiome changes [15]. The duration of hospitalization is also a wellknown risk factor for colonization with resistant bacteria $[16,17]$ because these patients are especially prone to colonization and subsequent infection. We found no significance difference between CRKP and CSKP patients regarding hospitalization in the previous 3 months, which differed from current epidemiology data and the CRKP infection spread pattern. By staying in the ICU with mechanical ventilation, patients have more chances for CRKP BSI. This finding apparently reflects prolonged hospitalization and poor functional status of patients, as shown by other studies $[18,19]$. These patients with mechanical ventilation and cumulative exposure to antibiotics likely have more opportunities for acquiring CRKP than patients without mechanical ventilation.

CRKP BSI seems to have high potential for spreading among patients in a health care facility and also by "cycling" of patients between institutions in the same region. This observation can be explained by study populations between the ICU and the whole hospital.

The attributable mortality was defined as the difference in allcause deaths between patients with CRKP infections and those with CSKP infections. In our study, the attributable mortality for CRKP BSI was $25.9 \%$ over 4 weeks. The high attributable mortality we found associated with CRKP BSI is consistent with previous studies, reporting crude mortality rates of $26 \%$ to $44 \%$ $[20,21]$. Indeed, the infectious event is likely to be a major contributor to death. However, attributable mortality from retrospective cohort studies has been criticized as to whether all excess deaths among cases should be attributed to infection [22]. The difference in the rates of crude and attributable mortality between 4 weeks and in-hospital endpoints in our study suggested that some deaths occur long after hospital admission and the onset of bacteremia. The drawback of crude mortality is its failure to identify related death. Nevertheless, the most important limitation of our study was not being able to follow the trajectory of illness during hospitalization.

In clinical practice, intensive care unit (ICU) setting has been traditionally considered the tip of the iceberg of hospital antimicrobial resistance. Although numerous hospital-based studies have suggested several comorbidities risk factors for the development of $K$. pneumoniae bacteremia, including diabetes mellitus, cancer, chronic liver disease, and biliary disease, we also found that the risk CRKP BSI associated with mechanical ventilation was higher compared with CSKP similar to EUROBACT International Cohort Study reported evidence [19]. To the best of our knowledge, our observational studies cannot provide proof of a causal relationship between mechanical ventilation and outcome. Possible reason refer to the severe condition for patient with mechanical ventilation.

Trend of increase in CRKP patients over the period of 2010 to 2016 in our hospital. The high burden of disease in the case of resistant pathogens is especially evident with the increase in CRKP and requires a wide range of control measures to account for the spread of these infections [20]. Prudent use of antibiotics, reflected also in the choices of combination antimicrobial therapy with agents exhibiting synergy, as well as efficient in hospital control practices including meticulous hand hygiene, are needed to control the spread of CRKP within hospitals [16,20-24]. Also, continued and efficient surveillance through adequate source control and antibiotic therapy is needed to stay up-to-date with the development and spread of the CRKP infections [25]. The high resistance rates found in contemporary study should encourage health authorities to preserve one of our most important resources, namely antimicrobials [19].

\section{Limitations}

Our study has several limitations. First, this was a singlecenter retrospective study with the inherent limitations of such a study design. Second, the sample was small without a matched cohort. Third, the impact of different and limited antibiotic options on outcome could not be further evaluated.

\section{Conclusion}

In conclusion, the CRKP BSI rates in our hospital in China are not similar to those in other countries. ICU stay is the highest area of infection and infection spread must be controlled. Future studies are needed regarding optimal antimicrobial stewardship interventions.

\section{References}

1. Munoz-Price LS, Poirel L, Bonomo RA (2013) Clinical epidemiology of the global expansion of Klebsiella pneumoniae carbapenemases. Lancet Infect Dis 13: 785-796. 
2. Dautzenberg MJ, Wekesa AN, Gniadkowski M (2015) The association between colonization with carbapenemase-producing enterobacteriaceae and overall ICU mortality: An observational cohort study. Crit Care Med 43: 1170-1177.

3. Patel G, Huprikar S, Factor SH, Jenkins SG, Calfee DP (2008) Outcomes of carbapenem-resistant Klebsiella pneumoniae infection and the impact of antimicrobial and adjunctive therapies. Infect Control Hosp Epidemiol 29: 1099-1106.

4. Yigit H, Queenan AM, Anderson GJ (2001) Novel carbapenemhydrolyzing beta-lactamase, KPC-1, from a carbapenem-resistant strain of Klebsiella pneumoniae. Antimicrob Agents Chemother 45 1151-1161.

5. Trecarichi EM, Tumbarello M (2014) Antimicrobial-resistant Gramnegative bacteria in febrile neutropenic patients with cancer: Current epidemiology and clinical impact. Curr Opin Infect Dis 27: 200-210.

6. Trecarichi EM, Pagano L, Martino B (2016) Bloodstream infections caused by Klebsiella pneumoniae in onco-hematological patients: Clinical impact of carbapenem resistance in a multicentre prospective survey. Am J Hematol 91: 1076-1081.

7. Giacobbe DR, Del Bono V, Trecarichi EM (2015) Risk factors for bloodstream infections due to colistin-resistant KPC-producing Klebsiella pneumoniae: Results from a multicenter case-controlcontrol study. Clin Microbiol Infect 21: 1106 e1-e8.

8. Tumbarello M, Trecarichi EM, Tumietto F (2014) Predictive models for identification of hospitalized patients harboring KPC-producing Klebsiella pneumoniae. Antimicrob Agents Chemother 58: 3514-3520.

9. Giannella M, Trecarichi EM, De Rosa F (2014) GRisk factors for carbapenem-resistant Klebsiella pneumoniae bloodstream infection among rectal carriers: a prospective observational multicentre study. Clin Microbiol Infect 20: 1357-1362.

10. Tamma PD, Goodman KE, Harris AD (2017) Comparing the outcomes of patients with carbapenemase-producing and noncarbapenemase-producing carbapenem-resistant enterobacteriaceae bacteremia. Clin Infect Dis 64: 257-264.

11. Ben-David D, Kordevani R, Keller N (2012) Outcome of carbapenem resistant Klebsiella pneumoniae bloodstream infections. Clin Microbiol Infect 18: 54-60.
12. Daikos GL, Petrikkos P, Psichogiou M (2009) Prospective observational study of the impact of VIM-1 metallo-betalactamase on the outcome of patients with Klebsiella pneumoniae bloodstream infections. Antimicrob Agents Chemother 53: 1868-873.

13. Cobo J, Morosini MI, Pintado V (2008) Use of tigecycline for the treatment of prolonged bacteremia due to a multiresistant VIM-1 and SHV-12 beta-lactamase-producing Klebsiella pneumoniae epidemic clone. Diagn Microbiol Infect Dis 60: 319-322.

14. Messina JA, Cober E, Richter SS (2016) Hospital readmissions in patients with carbapenem-resistant Klebsiella pneumoniae. Infect Control Hosp Epidemiol 37: 281-288.

15. Garrett WS (2015) Cancer and the microbiota. Science 348: 80-86.

16. Horcajada JP, Martinez JA, Alcon A (2006) Acquisition of multidrug-resistant Serratia marcescens by critically ill patients who consumed tap water during receipt of oral medication. Infect Control Hosp Epidemiol 27: 774-777.

17. Haverkate MR, Derde LP, Brun-Buisson C, Bonten MJ, Bootsma MC (2014) Duration of colonization with antimicrobial-resistant bacteria after ICU discharge. Intensive Care Med 40: 564-571.

18. Daikos GL, Markogiannakis A, Souli M, Tzouvelekis LS (2012) Bloodstream infections caused by carbapenemase-producing Klebsiella pneumoniae: A clinical perspective. Expert Rev Anti Infect Ther 10: 1393-1404.

19. Bogan C, Kaye KS, Chopra T (2014) Outcomes of carbapenemresistant Enterobacteriaceae isolation: Matched analysis. Am J Infect Control 42: 612- 620.

20. Hoxha A, Karki T, Giambi C (2016) Attributable mortality of carbapenem-resistant Klebsiella pneumoniae infections in a prospective matched cohort study in Italy, 2012-2013. J Hosp Infect 92: 61-66.

21. Falagas ME, Tansarli GS, Karageorgopoulos DE, Vardakas KZ (2014) Deaths attributable to carbapenem-resistant Enterobacteriaceae infections. Emerg Infect Diseases 20: 1170-1175.

22. Rello J (1999) Impact of nosocomial infections on outcome: Myths and evidence. Infect Control Hosp Epidemiol 20: 392-394. 